\title{
Psicologia jurídica e tomada de decisão em situações envolvendo abuso sexual infantil
}

\author{
Cátula Pelisoli1 - Universidade Federal do Rio Grande do Sul, Porto Alegre, Brasil \\ Lara Lages Gava - Universidade Federal do Rio Grande do Sul, Porto Alegre, Brasil \\ Débora Dalbosco Dell'Aglio - Universidade Federal do Rio Grande do Sul, Porto Alegre, Brasil
}

\begin{abstract}
Resumo
Este trabalho apresenta uma revisão teórica sobre a tomada de decisão em situações de alegação de abuso sexual infantil. Profissionais de saúde mental em todo o mundo necessitam tomar decisões acerca da veracidade dessas alegações, sendo muitas julgadas erroneamente. Este artigo apresenta o abuso sexual como um problema mundial, destaca as relações entre a psicologia e a justiça e aborda estudos que demonstram as influências de variáveis como vieses cognitivos, crenças prévias e gênero sobre as decisões tomadas por profissionais que avaliam tais casos. É fundamental que os profissionais reconheçam essas variáveis para que erros sobre esses julgamentos possam ser evitados e para que se aumente a qualidade das avaliações psicológicas forenses. A necessidade de experiência e conhecimento científico é abordada como possível contribuinte para a qualificação dos psicólogos que atuam na área e é sugerida a realização de pesquisas sobre o tema no contexto brasileiro.

Palavras-chave: Abuso sexual, Avaliação psicológica, Psicologia jurídica, Tomada de decisão.
\end{abstract}

Forensic psychology and decision making in situations involving child sexual abuse

\begin{abstract}
This paper presents a theoretical review on decision making in situations of alleged child sexual abuse. Mental health professionals around the world need to make decisions about the veracity of these claims, many being wrongly judged. This article presents sexual abuse as a global problem, highlights the relationship between psychology and justice, and approaches studies that demonstrate the influences of variables such as cognitive biases, prior beliefs and gender on the decisions taken by professionals who assess these cases. It is essential that professionals recognize these variables so that errors on these trials could be avoided and the quality of forensic psychological assessments could be increased. The need for experience and scientific knowledge is discussed as a possible contributor to the qualification of psychologists working in this area and researches on this subject within the Brazilian context are suggested.

Keywords: Sexual abuse, Psychological assessment, Forensic psychology, Decision-making.
\end{abstract}

Em 2004, a Organização Mundial de Saúde conceituou o abuso sexual infantil como uma emergência de saúde silenciosa e o considerou como a mais cruel e trágica infração aos direitos da criança à saúde e proteção (WHO, 2004). O abuso sexual infantil é definido como

o envolvimento da criança em uma atividade sexual que ela não compreende totalmente, para a qual ela não é bábil para dar consentimento, ou para a qual ela não está preparada em termos desenvolvimentais ou ainda que viola leis e tabus da sociedade. (WHO, 2004, p. 1)

Para Finkelhor (2009), abuso sexual é todo o espectro de crimes e ofensas sexuais de que crianças até 17 anos são vítimas, incluindo violências onde $\mathrm{o}$ perpetrador é parente ou desconhecido, adulto ou jovem.

O impacto do abuso sexual sobre a saúde física e psicológica é inquestionável, com evidências de possíveis consequências negativas apresentadas em muitos estudos. Uma metanálise demonstrou que, em

${ }^{1}$ Endereço para correspondência: Rua Ramiro Barcelos, 2.600/115 - 90035- 003 - Porto Alegre-RS.

E-mail: catulapelisoli@yahoo.com.br mais de nove mil vítimas de abuso investigadas em 37 estudos, transtorno de estresse pós-traumático, depressão, suicídio, promiscuidade sexual e prejuízo no desempenho acadêmico foram efeitos substanciais do abuso sexual infantil (Paolucci, Genius \& Violato, 2001). Mas ainda outros resultados negativos dessa violência já foram relatados: sentimentos crônicos de medo e ansiedade, pesadelos, dores de estômago e cefaleia, uso de álcool e drogas, problemas de comportamento, condutas ilegais, sentimentos de culpa, comportamento hipersexualizado, isolamento, sentimentos de desamparo e ódio, fugas de casa, baixa autoestima e agressividade, dentre outros sintomas (Amazarray \& Koller, 1998; Kendall-Tackett, Williams, \& Finkelhor, 1993; Myers, 2006; Nurcombe, 2000; Tyler, 2002). Trata-se, portanto, de uma violência que pode trazer consequências deletérias para a vida de suas vítimas, tanto em curto quanto em longo prazo, prejudicando seus funcionamentos psicossociais, relacionais, afetivos, cognitivos e acadêmicosocupacionais.

Crianças e adolescentes em todo o mundo, tanto do sexo feminino quanto masculino, são vítimas desse tipo de violência. Entretanto, as crianças do sexo feminino estão entre as principais vítimas (Finkelhor, 2009) dessa violência, que ocorre, na maior parte dos 
casos, dentro do próprio lar, perpetrado por um parente, consanguíneo ou não, mas com relações afetivas significativas. Em se tratando de violência intrafamiliar, que é o mais comum nos casos de abuso sexual (Finkelhor, 2009), sabe-se que, por serem dependentes, as crianças são aquelas que se encontram em maior situação de vulnerabilidade familiar e, por isso, correm mais risco (Maciel \& Cruz, 2009). Se o abuso sexual já é cercado por uma cultura de silêncio e estigma, isso é ainda mais evidente quando ocorre no santuário da própria casa e cometido por alguém que a criança conhece e em quem confia (WHO, 2004). Trata-se de uma violação dos direitos sexuais e humanos, realizada por meio de relações assimétricas permeadas pelo poder (econômico, geracional, de gênero) de um adulto sobre uma criança (Conselho Federal de Psicologia, 2009).

Em todo o cenário mundial, a preocupação crescente com o tema da violência intrafamiliar contra crianças e adolescentes é legitimada por dados que alarmam a sociedade e motivam políticas e programas de intervenção (Maciel \& Cruz, 2009). Apesar de parecer haver uma tendência de diminuição do abuso sexual infantil (Finkelhor, 2009; McGee, Garavan, Byrne, O’Higgins \& Conroy, 2010), esse fenômeno permanece sendo relativamente comum ao redor do mundo. Na Irlanda, por exemplo, foi conduzido um survey que demonstrou que quase um quarto dos homens $(24 \%)$ e quase um terço das mulheres $(30 \%)$ sofreram algum grau de abuso sexual na infância (McGee, Garavan, Byrne, O’Higgins \& Conroy, 2010). $\mathrm{Na}$ Austrália, alta prevalência foi também encontrada em uma grande amostra da população geral: $14 \%$ das mulheres e $7 \%$ dos homens relataram ter sofrido alguma forma de abuso sexual na infância (Moore, Romaniuk, Olsson, Jayasinghe, Carlin \& Patton, 2010). $\mathrm{Na}$ África, a situação é ainda mais dramática. Além de ser considerada como uma arma de guerra, a violência sexual também ocorre porque muitos acreditam no equívoco de que ter relações sexuais com uma virgem significa a cura do HIV/AIDS (International Committee of Red Cross - ICRC, 2006, 2008; WHO, 2004).

No Brasil, o Serviço Nacional de Denúncias pelo Telefone, coordenado pela Secretaria Especial dos Direitos Humanos do governo federal, Denúncia Nacional, o Disque 100, completou seis anos de atendimento, com mais de dois milhões de atendimentos e com cem mil denúncias registradas (Brasil, 2009). A procura pelo serviço tem aumentado a cada ano, tendo fechado o primeiro semestre de 2009 com 94 denúncias por dia. Desde o início dos atendimentos, em maio de 2003, os números totais indicam a região nordeste do país como a que mais denunciou e a região norte como a que menos denunciou. Entretanto, proporcionalmente, por número de habitantes, é a região centro-oeste que oferece a maior parte das denúncias. Do total de denúncias recebidas até maio de 2009, 31\% referiam-se à violência sexual $(58,5 \%$ de abuso sexual; $39,7 \%$ de exploração sexual; $1,6 \%$ de pornografia e $0,7 \%$ de tráfico de crianças e adolescentes). No que se refere aos dados de gênero, $81 \%$ das vítimas de violência sexual eram do sexo feminino (Brasil, 2009). Somente em 2010, de janeiro a junho, o Disque Denúncia realizou mais de 70 mil atendimentos, recebendo e encaminhando mais de 13 mil denúncias de todo o país, com uma média de 403 atendimentos e 73 denúncias ao dia. Cerca de $36 \%$ das denúncias desse ano referem-se a violência sexual, com pessoas do sexo feminino preponderantemente sendo as vítimas mais comuns (59\%) (Brasil, 2010).

O tema da violência sexual, por sua complexidade, perpassa diferentes áreas e disciplinas: a vítima necessita de atenção médica, psicossocial, jurídica e, também, dos profissionais da educação (Centro Regional de Atenção aos Maus-Tratos na Infância do ABCD, 2009). A psicologia, por sua vez, tem atuado neste tema a partir de diferentes vieses teóricos assumidos pelos profissionais e também de diferentes contextos onde estes se inserem. Por sua diversidade teórica e prática, a psicologia não apresenta ao problema do abuso sexual infantil uma conduta única, padrão ou alguma perspectiva consensual, mas um leque de possibilidades de entendimento do fenômeno e também de abordá-lo junto à criança vítima e sua família. Um exemplo disso é que a chance de encontrar dois psicólogos clínicos com a mesma abordagem para o problema é relativamente pequena. Se um mesmo paciente comparecer a dois consultórios de psicologia, ele pode encontrar diferentes maneiras de ver o mesmo problema. Na psicologia clínica, apesar das históricas oposições teóricas, essas divergências e diferenças não parecem ocasionar um problema para o paciente, na medida em que ambas as formas de tratar um paciente devem trazer resultados positivos para ele, cada uma com sua especificidade. Entretanto, quando se trata do contexto jurídico, diferentes abordagens entre psicólogos podem gerar confusões e dificuldades nas situações envolvendo abuso sexual infantil: uma entrevista conduzida de uma determinada forma ou um instrumento analisado sob determinada teoria pode gerar hipóteses divergentes entre psicólogos.

Diversas questões que envolvem a interlocução entre direito e psicologia têm sido investigadas nos meios acadêmicos. Os temas que se 
destacam nos periódicos científicos e nas publicações bibliográficas brasileiras, segundo uma revisão sistemática realizada por Pilatti e Silvino (2009), são: (a) critérios para avaliação de famílias que pleiteiam adotar crianças; (b) escuta da criança em processos judiciais; (c) papel do psicólogo na violência familiar contra crianças; (d) atendimento de mulheres agredidas em estágio supervisionado; (e) visão dos filhos em famílias pós-divórcio; (f) subjetividade de encarcerados do sistema prisional brasileiro; (g) parecer psicológico na justiça da infância e juventude; (h) destituição de poder familiar em processo de adoção; e (i) trabalho do agente de segurança penitenciária.

Especificamente no que diz respeito ao abuso sexual contra crianças e adolescentes, o diálogo entre psicologia e direito é de fundamental importância. É a partir desse diálogo e das relações que se estabelecem entre essas duas disciplinas que muitas vítimas terão acesso à garantia dos seus direitos de proteção e de saúde. $O$ problema da violência sexual exige a intersecção entre diferentes disciplinas e o trabalho conjugado dos profissionais, na forma da interdisciplinaridade. Diante de uma alegação de abuso sexual, a criança ou adolescente supostamente vítima deve passar por diferentes etapas: investigação da suspeita, decisão da agência que recebe a denúncia de ser ou não um caso de abuso, medidas de proteção para a criança e ação legal (Oates, Jones, Denson, Sirotnak, Gary \& Krugman, 2000). Especificamente, a psicologia pode atuar nessas diferentes etapas e com propósitos diversos. Este artigo abordará os aspectos relacionados ao abuso sexual no contexto da psicologia jurídica.

\section{Psicologia jurídica e abuso sexual infantil}

Segundo Myra y Lopez (2000), a psicologia jurídica é psicologia aplicada ao melhor exercício do direito. Trata-se, portanto, de um ramo da Psicologia que pretende auxiliar o direito, tendo em vista o objeto de estudo em comum, que é o homem, em sua complexidade e com seus conflitos. Este ramo se constituiu um espaço complexo, produto da interdisciplinaridade, que se faz essencial para garantir a justiça (Trindade, 2004). As relações entre essas disciplinas não têm se dado da forma mais harmônica possível, uma vez que existem diferenças no modo de olhar esse objeto de estudo. Enquanto a psicologia busca a compreensão do comportamento humano, o direito busca prescrever comportamentos; enquanto a psicologia pertence ao mundo do "ser", o direito pertence ao mundo do "dever-ser"; a psicologia fala de probabilidades, o direito tenta ser certeiro; a psicologia é descritiva e o direito é prescritivo (Huss, 2011). Dessa forma, fica clara a necessidade e relevância de um espaço comum de interlocução, que se dará a partir de relações mais próximas entre essas duas áreas, através dos papéis exercidos por cada um dos profissionais que se aventuram nesse campo.

Nos últimos anos, houve um avanço considerável das relações entre a psicologia e o sistema de justiça. Especificamente quando se trata de crimes em que a vítima é criança ou adolescente e em que não há, muitas vezes, evidências físicas que comprovem o fato, os operadores da lei vêm demandando questionamentos para a psicologia (Stein, Pergher \& Feix, 2009). Na medida em que estes operadores reconhecerem os subsídios que a psicologia tem a oferecer e que os psicólogos possam responder a essas demandas de forma substanciada e com embasamento científico, mudanças no sistema legal poderão ocorrer, bem como uma efetiva proteção de crianças e adolescentes testemunhas (Stein e Cols., 2009). O que de mais elementar o sistema de justiça pode fazer é aumentar a detecção e revelação dos casos de abuso sexual e a probabilidade de que os agressores sejam presos e processados (Finkelhor, 2009). O abuso sexual é um problema que envolve diferentes disciplinas, e, portanto, faz-se necessária a interlocução entre os diversos campos do saber, especialmente da psicologia e do direito. Nos casos em que a psicologia é chamada ao contexto judiciário, a obtenção de informações por meio da palavra da vítima tem o foco nas situações que ocorreram, que é exatamente o que faz diferença para a resolução do processo judicial (Rovinski \& Stein, 2009). Nas situações de abuso sexual infantil, a importância da psicologia se faz muito mais no sentido de confirmar a ocorrência da violência do que avaliar os impactos sofridos pela criança ou adolescente (Rovinski \& Stein, 2009).

A relação entre essas duas áreas - Psicologia e Direito - está em ascensão no Brasil, mas ainda há importante falha na formação dos profissionais, fazendo com que alguns busquem qualificação específica na pós-graduação ou formação complementar, quando esses cursos são oferecidos (Lago \& Bandeira, 2009). Faz-se necessário aos profissionais que atuam nesses temas conhecerem terminologias e obterem conhecimentos específicos, considerando os diferentes aspectos envolvidos no problema (Lago, Amato, Teixeira, Rovinski \& Bandeira, 2009). A psicologia jurídica vem crescendo e com ela cresce também a necessidade de qualificação para que os profissionais possam efetivamente auxiliar a Justiça, contribuindo, assim, para com aqueles envolvidos no processo: a criança vítima e sua família. Rovinski (2009) afirma que essa área é uma das mais 
promissoras dentro da psicologia no Brasil e que a atual falta de formação tem repercussões tanto na realização das avaliações forenses quanto para a proposição de novas intervenções.

$\mathrm{Na}$ medida em que a psicologia se instrumentaliza para atender às demandas do setor judiciário, ela contribui para a proteção da vítima e para seu desenvolvimento psicossocial. Essa instrumentalização inclui a qualificação profissional específica, com treinamentos e cursos dentro das áreas de interesse e de necessidade. Para alguns autores, a psicologia jurídica já é uma área consolidada de atuação do psicólogo brasileiro (Rovinski \& Cruz, 2009). Porém, segundo Cesca (2004), ainda é necessário configurar-se o lugar do psicólogo que trabalha neste contexto. Para a autora, qualificação e parceria entre profissionais são necessidades para que essa melhor configuração se efetive. A falta de preparo dos profissionais durante a formação acadêmica é evidente no nosso país, na medida em que são raros os cursos de graduação que oferecem disciplinas específicas de psicologia jurídica, sejam curriculares sejam eletivas (Rovinski, 2009).

Um dos principais papéis que o psicólogo pode exercer nessa área é o de perito. O psicólogo perito que atua em casos de abuso sexual, além de muitas vezes apresentar falta de qualificação para o exercício dessa atividade, pode apresentar uma formação muito diversificada, tendo desde apenas a graduação em curso universitário até o nível de doutorado (Klettke, Graesser \& Powel, 2010). Os casos de abuso sexual são permeados de dúvidas e incertezas, histórias complexas e dinâmicas difíceis, que muitas vezes impedem que um diagnóstico da situação seja consubstanciado. Em estudo realizado por Oates, Jones, Denson, Sirotnak, Gary e Krugman (2000), foi verificado que $42,8 \%$ dos casos de uma agência de proteção que recebe casos de abuso sexual infantil nos Estados Unidos foram considerados com respaldo, enquanto $21,1 \%$ foram considerados inconclusivos, $33,6 \%$ foram considerados não sendo de fato abuso sexual e 2,5\% como referências erradas de abuso sexual. Este estudo demonstra a importância de que sejam realizados diagnósticos mais precisos dessas situações.

$\mathrm{Na}$ literatura, a perícia tem sido definida como a habilidade para identificar, integrar e extrair conclusões razoavelmente corretas de dados multidimensionais e complexos, sem que sejam afetados por conhecidos vieses e ilusões (FinnilãTuohimaa, Santtila, Sainio, Niemi \& Sandnabba, 2009). Para isso, não basta apenas o conhecimento especializado, mas também são necessárias boas habilidades para a tomada de decisões. Nos casos de violência sexual, os investigadores têm que obter e integrar matérias muitas vezes contraditórias, uma vez que não há um indicador específico que possa determinar se houve abuso sexual ou não. Em muitos casos de alegação de abuso sexual infantil (ASI), a única evidência disponível é o testemunho da criança e, em todos os casos, é crucial que uma descrição confiável da alegada vítima seja obtida (FinnilãTuohimaa e Cols., 2009).

Para que esta descrição confiável seja obtida, é necessário que o perito que está avaliando o caso tenha conhecimento dos fatores que podem fazer com que uma entrevista seja sugestiva e habilidade para evitar questões que possam sugestionar crianças e adolescentes (Finnilã-Tuohimaa e Cols., 2009). Entretanto, estudos sugerem que clínicos investigando ASI fazem uso de questões sugestivas numa alarmante proporção. Finnilã-Tuohimaa e colegas (2009) identificaram que profissionais que investigam ASI (psiquiatras, psicólogos e assistentes sociais), ao se depararem com entrevistas transcritas de outros profissionais que realizaram entrevistas de avaliação com crianças, identificaram perguntas sugestivas. Entretanto, esses profissionais não se mostraram da mesma maneira sensíveis a outros componentes que podem influenciar a entrevista, como presença de ameaças e recompensas, que podem fazer com que o entrevistado decida por não abordar determinados assuntos (quando algum tipo de ameaça por parte do entrevistador se coloca) ou a investir e desenvolver algum tópico (quando recompensado). Esses autores observaram também que, quando não há transcrição da entrevista, os profissionais tendem a confiar que seus colegas não fizeram uso de questões sugestivas e acabam por não considerar isso como uma preocupação. Um importante resultado encontrado neste estudo foi uma associação positiva entre experiência clínica, competência científica e perícia autoavaliada com a sensibilidade para perguntas sugestivas. Dessa forma, fica demonstrado que quanto mais experiência e competência tem o profissional, mais ele percebe a sugestionabilidade de entrevistas com crianças.

Granjeiro e Costa (2008) realizaram um estudo de caso envolvendo uma situação de abuso sexual de um pai contra duas filhas, a partir da qual diferentes atores envolvidos no processo jurídico fizeram suas reflexões. Um juiz, uma promotora de justiça, uma defensora pública, uma psicóloga e uma assistente social deram entrevistas sobre o papel do estudo psicossocial forense nesses casos. Essas entrevistas indicaram que os documentos elaborados pela 
psicologia são considerados pelos operadores do direito como prova e que, quando apresentam detalhes da violência, parecem ser importantes na acusação criminal. Além disso, esses documentos parecem suprir a insuficiência do magistrado quanto ao conhecimento técnico, propiciando maior certeza fática. Nesse estudo, as autoras mostram que o olhar da psicologia diante desses casos revela duas vertentes, uma psicossocial, de oferecer ajuda e reflexão, e outra de cunho investigativo-policial, que está comprometida com a verificação dos fatos. Tendo por base essa investigação, o estudo também mostrou que uma maior articulação entre essas duas áreas e maior consistência na relação entre elas é uma necessidade premente. Um contraponto disso seria o artigo de Cesca (2004), que informa que os laudos de avaliação psicológica costumam ser desprezados nos tribunais, porque estes não contemplam provas materiais da violência. Essa autora aborda ainda a questão de que existe na lei a presença da punição para o autor do crime, mas não a reabilitação da vítima; e de que o sistema não contempla nem o acompanhamento do abusado, tampouco do abusador.

Nas situações de abuso sexual infantil, o papel de um perito psicólogo é de grande relevância, especialmente porque nesses casos frequentemente faltam evidências físicas ou médicas que comprovem o fato. A frequência com que psicólogos têm sido chamados a atuarem como peritos tem aumentado ao redor do mundo. Peritos psicólogos são procurados para incrementar a credibilidade do depoimento da vítima ou prover os jurados com informação que pode ajudá-los a decidir se a criança foi de fato abusada (Klettke, Graesser \& Powel, 2010). Por se tratar de um tema complexo, a preocupação com a acurácia de uma avaliação forense tem motivado estudos que investigam diferentes fatores que influenciam a tomada de decisão tanto por psicólogos (Kovera, Borgida, Gresham, Swim \& Gray, 1993) como por possíveis jurados (Pozzulo, Dempsey, Maeder \& Allen, 2010).

É bem documentado que não há uma síndrome específica do abuso sexual e de que muitas vítimas não apresentam sintomas específicos (Dammeyer, 1998). Mais difícil ainda é diferenciar vítimas de abuso sexual de vítimas de outras formas de abuso e negligência. Autores têm sugerido, por esse motivo, avaliações compreensivas no lugar de avaliações de indicadores específicos de abuso. Já que indicadores isolados de abuso são raramente definitivos, faz sentido que examinadores investiguem múltiplos indicadores antes de chegar a conclusões. Avaliações de ASI geralmente envolvem dois aspectos: a identificação da ocorrência de abuso no passado e a predição de possíveis abusos futuros (se a criança está em risco). Para alcançar esses objetivos, as melhores fontes de informação são os exames médicos e os relatos da criança (Dammeyer, 1998).

É necessário que os profissionais identifiquem e usem as melhores informações disponíveis quando formulam suas decisões acerca da ocorrência ou não do abuso sexual. Ao mesmo tempo, eles devem manter consciência das limitações de seus métodos. Parte da dificuldade existe porque os profissionais que conduzem avaliações desse tipo representam disciplinas diferentes, como saúde mental, lei, proteção da criança e medicina. $O$ treinamento e experiência desses profissionais são variados e, por isso, é de se esperar que seus métodos de investigação também o sejam (Dammeyer, 1998). Num survey conduzido com profissionais que avaliam situações de ASI, os mais frequentes procedimentos e/ou indicadores de abuso foram operacionalizados como: (a) exames médicos; (b) relato da criança sobre a ocorrência do abuso; (c) informações obtidas a partir de procedimentos de avaliação incluindo bonecos, desenhos, fantoches, e casa de bonecas; (d) presença-ausência de comportamento hipersexualizado não-apropriado; e (e) dados obtidos de testes psicológicos aplicados na criança e no abusador alegado. É importante observar que a informação obtida baseada nesses procedimentos pode variar amplamente entre avaliadores, bem como entre diferentes grupos de profissionais (Dammeyer, 1998).

\section{Tomada de decisão em situações de abuso sexual infantil}

As decisões sobre as situações de abuso sexual envolvem diferentes profissionais, passando por operadores da lei e profissionais como assistentes sociais e psicólogos. Apesar de serem proferidas pela figura do juiz, as decisões dependem da participação de outros personagens, como o promotor, que oferece a denúncia, por exemplo. No que se refere ao papel das disciplinas psicossociais nessas situações, como o serviço social e a psicologia, sabe-se da importância de seus estudos para a tomada de decisão pelos operadores da lei. Mesmo sendo a decisão última e com valor de lei aquela do juiz, uma decisão clínica é realizada por esses profissionais que avaliam o caso. Nesta seara, o que se tem encontrado em diferentes estudos é a predominância de discordâncias entre avaliadores, como pode ser visto na extensa revisão realizada por Herman (2005). Para esse autor, provavelmente milhares de decisões errôneas sobre alegações de abuso sexual infantil são feitas a cada ano.

Herman (2005) realizou uma revisão de inúmeros estudos que demonstram a baixa 
concordância entre avaliadores a respeito das mesmas situações de abuso sexual infantil. Um exemplo é o estudo de Realmuto e Wescoe (1992), em que 15 crianças foram entrevistadas por um psiquiatra infantil e seis delas foram classificadas como sexualmente abusadas. Entretanto, o experimentador havia realizado uma classificação prévia, que não obteve correlação com a avaliação psiquiátrica. Num segundo experimento desses mesmos autores, 13 vídeos de entrevistas foram vistos por 14 juízes clínicos, totalizando 182 julgamentos. Os 14 juízes demonstraram um baixo nível de concordância entre eles $(k=0,36)$ e a correlação entre essas classificações e as predeterminadas pelo experimentador foi insignificante $(=0,08)$. Assim como nesse estudo, outros experimentos revisados por Herman não obtiveram níveis relevantes de concordância, indicando que profissionais discordam muito quanto a decisões sobre se um abuso sexual ocorreu ou não.

Mesmo profissionais que não atuam diretamente com a Justiça, nesses casos tomam decisões quanto à veracidade da situação de abuso sexual infantil, seja para emitir documentos, seja para planejar intervenções, levantando hipóteses sobre a probabilidade de ocorrência do evento. Sabe-se que muitos casos não apresentam evidências factuais suficientes e, por tal motivo, os profissionais da saúde mental e especialistas em desenvolvimento infantil têm sido chamados aos tribunais (Finnilã-Tuohimaa, Santtila, Sainio, Niemi \& Sandnabba, 2005). Em se tratando de um crime emocionalmente carregado, há considerável pressão sobre os profissionais para que atuem no melhor interesse da criança. Entretanto, não são muitos os estudos que têm sido feitos para investigar os processos de tomada de decisão de profissionais que conduzem investigações de ASI (Finnilã-Tuohimaa e Cols., 2005). Levando-se em conta que as divergências entre os profissionais com relação às situações de abuso sexual são intensas (Herman, 2005), parece necessário entender melhor as influências que estão subjacentes ao processo de decidir sobre se uma alegação de abuso sexual é ou não uma realidade factual.

As pesquisas têm demonstrado que $\mathrm{O}$ julgamento humano sob incerteza não é baseado em probabilidades estatísticas, mas sim em regras heurísticas, o que em tarefas complexas pode levar a erros sistemáticos e que também pode afetar o trabalho clínico (Dammeyer, 1998). Heurísticas são compreendidas como regras gerais de influência que contribuem para as tomadas de decisões, com custo reduzido de tempo e esforços (Tonetto e Cols., 2006). Para tomar uma decisão a partir de uma questão incerta, as pessoas buscam soluções que tendem a satisfazer suas aspirações. Os seres humanos apresentam um processamento mental limitado, com vieses de memória e sem domínio da racionalidade. As pessoas têm capacidade limitada para o trabalho mental e utilizam de estratégias simplificadas para julgar alternativas: uma dessas estratégias é o uso de heurísticas (Tonetto e Cols., 2006). As heurísticas da representatividade e da disponibilidade podem se configurar como vieses cognitivos influenciando a tomada de decisão em situações de incerteza, que são características nos casos de abuso sexual contra crianças e adolescentes.

A heurística da representatividade pressupõe que o julgamento da probabilidade de um evento incerto é tomado de acordo com o quão similar ou representativo ele é da população da qual se origina e de acordo com o grau no qual ele reflete os aspectos proeminentes do processo pelo qual é gerado (Sternberg, 2000). Uma probabilidade alta de ocorrência é conferida a um evento quando este é típico ou representativo desse tipo de situação (Tonetto e Cols., 2006). Nos casos de abuso sexual, pode-se pensar que uma história contada de forma que inclua conteúdos já conhecidos por parte dos profissionais da área será mais facilmente considerada como verdadeira. Dessa forma, uma hipótese é a de que quanto mais itens representativos de situações de abuso sexual tiver uma história, maior caráter de verdade os psicólogos darão a ela. Entre os aspectos representativos dos casos de abuso sexual, destaca-se a presença de algumas características como o gênero feminino como o mais vitimizado, famílias reconstituídas, antecedentes criminais do réu, características de negligência e/ou depressão maternas, dinâmica de segredo, barganhas e ameaças (Brito, Zanetta, Mendonça, Barison \& Andrade, 2005; Deslandes, 1994; Flores \& Caminha, 1994; Narvaz, 2003; Sanderson, 2005; Santos \& Dell'Aglio, 2008).

Essa influência é também chamada de pensamento representativo, e pode ser verificada em situações nas quais os clínicos identificam um determinado sintoma e o compreendem como uma evidência de abuso sexual. Um exemplo frequente disso é a associação do comportamento hipersexualizado em crianças com a ocorrência de abuso, embora esse sintoma não possa ser considerado como uma prova definitiva de ASI. Para FinnilãTuohimaa e Cols. (2005), os profissionais combinam um certo problema com certas características e esquecem que é possível um caso apresentar as características, sem, contudo, ter ocorrido o problema. 
Outra influência sobre a tomada de decisão é entendida como heurística da disponibilidade, que é utilizada em julgamentos com base em quão facilmente podemos trazer à memória o que percebemos como circunstâncias relevantes de um fenômeno (Sternberg, 2000). A partir dessa perspectiva, profissionais podem exagerar a probabilidade de um fenômeno quando este é encontrado frequentemente ou quando é emocionalmente carregado. Dessa forma, aqueles clínicos que encontram muitos casos de alegação de abuso sexual tendem a superestimar a probabilidade do abuso (Finnilã-Tuohimaa e Cols., 2005).

Demonstrando a influência destas heurísticas, o estudo de Finnilã-Tuohimaa e colegas (2005) comparou três grupos de profissionais que investigam ASI: psiquiatras, psicólogos e assistentes sociais. Nesse estudo, os autores identificaram que a categoria profissional que detinha maior experiência em abuso sexual eram os psiquiatras, que se autoavaliaram também como tendo maior perícia ou especialização no assunto. Entretanto, seu conhecimento foi avaliado e verificou-se que a falta de conhecimento teórico é geral entre esses profissionais, incluindo os psiquiatras. Os autores concluíram que por terem mais experiência com abuso, os psiquiatras avaliaram a prevalência de forma mais alta, indicando então a influência da experiência anterior na tomada de decisão (disponibilidade). Este estudo mostrou que os profissionais confiam mais na sua experiência do que no conhecimento científico e teórico ao tomar decisões em investigações de ASI. Isto pode significar que quanto mais experiência com abuso sexual tem o profissional, mais ele tende a superestimar a probabilidade desse fenômeno ocorrer.

Outros fatores cognitivos influenciando a tomada de decisão em casos de ASI parecem ser crenças anteriores, em que o entrevistador apresenta um viés preexistente em direção a acreditar que o abuso tenha ocorrido de fato (Finnilã-Tuohimaa e Cols., 2005). Dois estudos podem contribuir para corroborar essa hipótese. Um deles foi realizado por Kovera, Borgida, Gresham, Swim e Gray (1993), em que profissionais membros da International Society for Traumatic Stress Studies - ISTSS participaram de um survey que investigou as crenças sobre abuso sexual infantil e sobre a capacidade da criança testemunha. A iniciativa dos autores foi decorrente das controvérsias a respeito de que muitos psicólogos atuavam como impassíveis defensores das crianças, em detrimento de outros papéis que eles poderiam exercer. Atuando dessa forma, os psicólogos podem ver abuso sexual quando este não existe e, assim, realizar julgamentos clínicos com pouca acurácia. Neste survey com 340 membros do ISTSS, as mulheres e os membros que trabalhavam com vítimas de abuso sexual infantil apresentavam crenças mais fortes em relação à credibilidade da criança do que homens e membros que trabalhavam com veteranos ou outros sofrimentos pós-traumáticos. Resultado similar foi obtido em um estudo com jurado simulado, no qual os autores encontraram que as mulheres estudantes universitárias avaliaram os níveis de credibilidade da vítima como mais altos do que o fizeram os jurados homens (Pozzulo, Dempsey, Maeder \& Allen, 2010). Além disso, os jurados homens avaliaram a credibilidade do réu em níveis significativamente mais altos do que as juradas mulheres. Outro resultado importante desse estudo foi o de que o gênero do júri teve um efeito significativo sobre a percepção de desejo da vítima para o crime e causação, mas não para responsabilidade, ou seja, os jurados homens deram níveis mais altos de responsabilidade para a vítima, comparados às mulheres. Isso significa que os homens, nesse estudo, consideraram que a vítima desejava e era causa do crime, mais do que as mulheres consideraram. Por sua vez, as juradas mulheres avaliaram os níveis de responsabilidade do réu significativamente mais altos do que os jurados homens. Portanto, esses estudos demonstram a influência do gênero na tomada de decisão sobre situações de abuso sexual infantil.

O gênero do profissional avaliador também se mostrou uma influência significativa no estudo de Finnilã-Tuohimaa e colegas (2005). Nesse trabalho, os participantes homens apresentaram crenças menos significativas do que as mulheres, principalmente no que se refere à defesa da criança. Uma explicação seria a de que as mulheres, em geral, tendem a ter opiniões mais extremas quando o problema se refere a crianças. Esse estudo corroborou a ideia de que os clínicos apresentam fortes crenças que podem influenciar sua tomada de decisão (Finnilã-Tuohimaa e Cols., 2005).

Pennington e Hastie (1988) desenvolveram um modelo explicativo sobre como as pessoas avaliam as evidências em um julgamento e, dessa avaliação, tomam suas decisões. Nesse modelo, intitulado "Modelo da História em Decisões Judiciais", os autores combinam três tipos de informações: (a) informações específicas do caso, dadas ao longo do julgamento; (b) conhecimento prévio sobre eventos similares ao que deve ser julgado; e (c) expectativas gerais sobre o que constitui uma história completa. Esse modelo indica que as pessoas constroem uma explanação na forma de narrativa para avaliar as evidências em um julgamento: elas constroem um modelo causal e baseiam neste suas subsequentes decisões. Um jurado pode construir mais de uma história, mas aquela que for mais coerente será 
usualmente mais aceita. A coerência integra os juízos de completude, consistência e plausibilidade. Consistência é a extensão em que a história não apresenta contradições, e a plausibilidade é avaliada pelas comparações das sequências dos eventos. Se mais de uma história for coerente, resultará a incerteza (Pennington \& Hastie, 1988).

Com esses estudos, evidencia-se o fato de que os profissionais não baseiam suas decisões apenas nos dados das entrevistas com as crianças e adolescentes. Se o pensamento representativo tem sido encontrado mesmo em estudos de competência do júri, fica evidente que, em muitos casos, os jurados utilizam-se de heurísticas e pensamentos subjetivos mais do que evidências objetivas para encontrar uma decisão para o caso: "É possível que os problemas na investigação do abuso sexual infantil originem-se de outros vieses do processo de tomada de decisão, além de entrevistas sugestivas" (Finnilã-Tuohimaa e Cols., 2009, p. 141).

\section{Considerações finais}

Os estudos que buscam compreender como processos psicológicos influenciam na tomada de decisão têm se situado numa área denominada psicologia da deliberação legal (Pilatti, Silvino, Miura \& Caldas, 2010). Este campo é carente de pesquisas empíricas no Brasil (Pilatti \& Silvino, 2009). Em se tratando do complexo tema do abuso sexual infantil, sabe-se que, na maioria dos casos, evidências físicas não são encontradas (Herman, 2010). Com essa ausência da materialidade, necessária para que os procedimentos jurídicos gerem a responsabilização do agressor e a proteção da vítima, uma grande responsabilidade recai sobre a psicologia: a de contribuir com a justiça, vítimas e suas famílias para o melhor andamento do caso. A avaliação psicológica constitui-se, nesse contexto, como uma das principais provas e, muitas vezes, única forma de prover o caso com algum conteúdo técnico-científico. Entretanto, as dificuldades da própria ciência psicológica e a complexidade do corpo teórico sobre o tema implicam problemas no que se refere à validade dessas avaliações. Ao mesmo tempo em que a sociedade torna-se consciente da importância da psicologia nesses casos, a ciência conhece suas limitações. Os erros são frequentes e, nessa área, podem ocasionar mudanças na vida de crianças e famílias e acarretar sofrimentos desnecessários (Herman, 2005). É uma responsabilidade social e científica dos psicólogos qualificarem-se para o exercício de avaliações de alta complexidade como estas.
Com base nos estudos revisados, fica claro que apenas uma entrevista não sugestiva e cuidadosa não garante que outras variáveis deixem de exercer suas influências. Crenças prévias parecem exercer um papel importante na medida em que alguns profissionais tendem a atuar muito mais como defensores da criança do que como avaliadores neutros (Kovera, Borgida, Gresham, Swim \& Gray, 1993). O gênero do profissional também aparece com uma influência significativa, uma vez que as mulheres tendem a avaliar de forma superior os níveis de credibilidade da vítima e os homens os do réu (Pozzulo, Dempsey, Maeder \& Allen, 2010). Além de crenças prévias e do gênero, a tomada de decisões em situações de incerteza tem seu processo característico, que inclui atalhos cognitivos (heurísticas), sujeitos a erros e vieses (FinnilãTuohimaa e Cols., 2009), e uma avaliação da coerência da história (Pennington \& Hastie, 1988). O fato de ter mais experiência em casos de abuso sexual e considerar características representativas como fortes indicadores de que o abuso de fato ocorreu parece, então, ser tanto uma necessidade quanto um risco. Uma necessidade porque se sabe que a experiência e o conhecimento sobre a tipicidade de uma questão psicológica é um fator relevante para os psicólogos, de um modo geral. É a experiência que faz com que os psicólogos tenham maior familiaridade com os processos psicológicos, que qualifiquem $\mathrm{o}$ seu trabalho $\mathrm{e}$ refinem $\mathrm{o}$ seu entendimento clínico. No entanto, por outro lado, a experiência pode ser um risco, na medida em que quanto mais experiência tiver, maior será a probabilidade do profissional pensar que um próximo caso também será verdadeiro. Além disso, se estivermos presos em "fórmulas" de indicadores de abuso, sem questionar a realidade factual e objetiva, tão valorizada pelo direito e muitas vezes desvalorizada pela psicologia, corremos um risco enorme de identificar ou não situações de abuso erroneamente, gerando dificuldades talvez insuperáveis para os envolvidos.

Além dessas variáveis que têm sido discutidas e percebidas nas situações de abuso sexual na literatura internacional, acredita-se que ainda outras, de ordem técnica e teórica, também possam representar vieses que influenciem diretamente decisões acerca dos casos de violência contra crianças e adolescentes. Dois exemplos dessas variáveis são a identificação teórica do profissional e a escolha dos instrumentos de avaliação. Pensa-se que esses fatores possam também influenciar o posicionamento do profissional para ter uma determinada decisão ou outra em relação ao diagnóstico da situação abusiva. A hipótese lançada aqui se refere ao fato de não haver um padrão ouro na 
avaliação do abuso sexual (Herman, 2005) e de o campo da psicologia estar longe de apresentar diretrizes comuns (Conselho Federal de Psicologia, 2009). A diversidade da psicologia, ao mesmo tempo em que é riqueza teórica e técnica, perde muitas vezes em padronização. Ressalta-se que padronização não é utilizada aqui como um "enquadramento", como algo fechado e rígido.

A solução para um problema tão complexo como este parece estar de fato muito distante. Entretanto, o caminho para isso parece ser a combinação entre experiência e conhecimento científico. Muitos profissionais não apresentam níveis adequados de treinamento, conhecimento e habilidades para realizar entrevistas altamente qualificadas com crianças ou para descrever os fatores que distinguem as verdadeiras das falsas acusações de abuso (Herman, 2005). A experiência profissional é frequentemente referida como um importante fator para aumentar a especialização/perícia e melhorar a tomada de decisão clínica em investigações sobre o ASI. Considerando essa relevância, alguns autores sugerem que em treinamentos com investigadores de ASI deve-se não apenas prover informações sobre prevalência e contexto do ASI e do incesto, mas também abordar os processos de tomada de decisão (Finnilã-Tuohimaa e Cols., 2009). Treinamentos futuros também devem incluir discussões sobre a influência que crenças prévias dos profissionais podem ter sobre o manejo e avaliação dos casos. Para evitar esses vieses, os clínicos devem estar conscientes de que crenças podem ter influência sobre as percepções de evidências, sobre a construção de uma sequência plausível de eventos, avaliação da credibilidade do acusado e da alegada vítima e também sobre o padrão de prova que eles requerem para se convencerem da culpa de uma pessoa acusada (FinnilãTuohimaa e Cols., 2005).

Além da responsabilidade de cada profissional em buscar conhecimento específico, cabe aos pesquisadores brasileiros a investigação dessas influências no contexto nacional, uma vez que não existem estudos sobre o tema no país. Investigações que procuram qualificar o trabalho do psicólogo nas avaliações de abuso sexual infantil, mesmo ainda sendo em pequeno número e restritas a alguns núcleos de pesquisa, parecem ter um espaço garantido na comunidade acadêmica. As falsas memórias, alienação parental e estudos sobre avaliações e instrumentos para uso nesses casos são extremamente relevantes e refletem as preocupações do pesquisador brasileiro em impulsionar o crescimento especializado da área. Entretanto, faltam estudos que investiguem aspectos do próprio avaliador, como os processos que ele utiliza para tomar decisões, as variáveis que exercem influências sobre isso e até mesmo sobre a formação e capacitação dele para a atuação neste tema. Revela-se, portanto, um campo de estudos e investigações pouco explorado no mundo e ausente das discussões brasileiras, que envolve por um lado, profissionais psicólogos que avaliam e atendem a casos de abuso sexual infantil e pesquisadores da área, e, por outro lado, crianças, adolescentes e famílias que são o objeto dessas investigações. Assim, é necessária a busca de conhecimento e qualificação continuados, não permitindo que possíveis erros técnicos atinjam bruscamente a população vítima desse tipo de violência.

\section{Referências}

Amazarray, M. R. \& Koller, S. H. (1998). Alguns aspectos observados no desenvolvimento de crianças vítimas de abuso sexual. Psicologia Reflexão e Critica, 11(3), 546-555.

Brasil (2009). Disque 100: cem mil denúncias e um retrato da violência sexual infanto-juvenil. Programa Nacional de Enfrentamento da Violência Sexual Contra Crianças e Adolescentes. Subsecretaria Nacional de Direitos da Criança e do Adolescente. Secretaria Especial de Direitos Humanos da Presidência da República. Acessado em 27 de julho de 2010. Disponível em http://www.bancadigital.com.br/sedh/reader2/?p $\mathrm{ID}=4$.

Brasil (2010). Disque Denúncia Nacional - DDN 100: relatório janeiro-junho 2010. Programa Nacional de Enfrentamento da Violência Sexual Contra Crianças e Adolescentes. Subsecretaria Nacional de Direitos da Criança e do Adolescente. Secretaria Especial de Direitos Humanos da Presidência da República. Acessado em 27 de julho de 2010. Disponível em http://www1.direitoshumanos.gov.br/spdca/expl oracao_sexual/Acoes_PPCAM/disque_denuncia

Brito, A. M. M., Zanetta, D. M. T., Mendonça, R. C. V., Barison, S. Z. P. \& Andrade, V. A. G. (2005). Violência doméstica contra crianças e adolescentes: estudo de um programa de intervenção. Ciência e Saúde Coletiva, 10(1), 143-149.

Centro Regional de Atenção aos Maus-Tratos na Infância do ABCD - CRAMI (2009). Violência sexual contra crianças e adolescentes: compreender para prevenir. 2. ed. Santo André, SP: CRAMI-ABCD. 
Cesca, T. B. (2004). O papel do psicólogo jurídico na violência intrafamiliar: possíveis articulações. Psicologia \& Sociedade, 16(3), 41-46.

Conselho Federal de Psicologia (2009). Serviço de proteção social a crianças e adolescentes vitimas de violência, abuso e exploração sexual e suas famílias: referências para a atuação do psicólogo. Brasilia: Conselho Federal de Psicologia.

Dammeyer, M. D. (1998). The assessment of child sexual abuse allegations: using research to guide clinical decision making. Behavioral Sciences and the Law, 16, 21-34.

Deslandes, S. F. (1994). Atenção a crianças e adolescentes vítimas de violência doméstica: análise de um serviço. Cadernos de Saúde Pública, 10(1), 177-187.

Finkelhor, D. (2009). The prevention of childhood sexual abuse. The Future of Children, 19(2), 169-194.

Finnilã-Tuohimaa, K., Santtila, P., Sainio, M., Niemi, P. \& Sandnabba, K. (2005). Connections between experience, beliefs, scientific knowledge and selfevaluated expertise among investigators of child sexual abuse in Finland. Scandinavian Journal of Psychology, 46, 1-10.

Finnilã-Tuohimaa, K., Santtila, P., Sainio, M., Niemi, P. \& Sandnabba, K. (2009). Expert judgment in cases of alleged child sexual abuse: clinicians' sensitivity to suggestive influences, pre-existing beliefs and base rates estimates. Scandinavian Journal of Psychology, 50, 129-142.

Flores, R. \& Caminha, R. (1994). Violência sexual contra crianças e adolescentes: algumas sugestões para facilitar o diagnóstico correto. Revista de Psiquiatria do Rio Grande do Sul, 16(2), 158-167.

Granjeiro, I. A. C. L. \& Costa, L. F. (2008). O estudo psicossocial forense como subsídio para a decisão judicial na situação de abuso sexual. Psicologia Teoria e Pesquisa, 24(2), 161-169.

Herman, S. (2005). Improving decision making in forensic child sexual abuse evaluations. Law and Human Behavior, 29(1), 87-120.

Herman, S. (2010). The role of corroborative evidence in child sexual abuse evaluations. Journal of Investigative Psychology and Offender Profiling, 7, 189212.

Huss, M. T. (2011). Psicologia forense: pesquisa, prática clínica e aplicações. Porto Alegre: Artmed.
International Committee of Red Cross - ICRC (2006). Congo Kinshasa: The hidden battle. Acessado em 21 de setembro de 2009. Disponível em http:/ /icrc.org/web/eng/siteeng0.nsf/htmlall/f00 917-v?opendocument.

International Committee of Red Cross - ICRC (2008). Confronting sexual violence as a method of warfare. Acessado em 21 de setembro de 2009. Disponível em http://icrc.org/Web/por/sitepor0.nsf/html/sexu al-violence-interview-260608.

Kendall-Tackett, K. A., Williams, L. M. \& Finkelhor, D. (1993). Impact of sexual abuse on children: a review and synthesis of recent empirical studies. Psychological Bulletin, 113, 164-180.

Klettke, B., Graesser, A. C. \& Powel, M. B. (2010). Expert testimony in child sexual abuse cases: the effects of evidence, coherence and credentials on juror decision making. Applied Cognitive Psychology, 24, 481-494.

Kovera, M. B., Borgida, E., Gresham, A. W., Swim, J. \& Gray, E. (1993). Do child sexual abuse experts hold pro-child beliefs? A survey of the International Society for Traumatic Stress Studies. Journal of Traumatic Stress, 6(3), 383-404.

Lago, V. M., Amato, P., Teixeira, P. A., Rovinski, S. L. R. \& Bandeira, D. (2009). Um breve histórico da Psicologia Jurídica no Brasil e seus campos de atuação. Estudos de Psicologia, 26(4), 483-491.

Lago, V. M., \& Bandeira, D. (2009). A psicologia e as demandas atuais do direito de família. Psicologia: Ciência e Profissão, 29(2), 290-305.

Maciel, S. K. \& Cruz, R. M. (2009). Violência psicológica contra crianças nas interações familiares: Problematização e diagnóstico. Em S. L. R. Rovinski \& R. M. Cruz (Eds.). Psicologia jurídica: perspectivas teóricas e processos de intervenção. São Paulo: Vetor.

McGee, H., Garavan, R., Byrne, J., O’Higgins \& Conroy, R. M. (2010). Secular trends in child and adult sexual violence - one decreasing and the other increasing: a population survey in Ireland. The European Journal of Public Health, 1-6.

Mira y Lopez, E. (2000). Manual de psicologia jurídica. Campinas: Péritas.

Moore, E. E., Romaniuk, H., Olsson, C. A., Jayasinghe, Y., Carlin, J. B. \& Patton, G. C. (2010). The prevalence of childhood sexual abuse and 
adolescent unwanted contact among boys and girls living in Victoria, Australia. Child Abuse \& Neglect, 34, 379-385.

Myers, J. E. B. (2006). Child protection in America: past, present and future. Nova Iorque: Oxford University Press.

Narvaz, M. G. (2003). Quem são as mães das vítimas de incesto? Nova Perspectiva Sistêmica, 21, 40-44.

Nurcombe, B. (2000). Child sexual abuse I: psychopathology. Australian and New Zealand Journal of Psychiatry, 34(1), 85-91.

Oates, R. K., Jones, D. P. H., Denson, D., Sirotnak, A., Gary, N. \& Krugman, R. D. (2000). Erroneos concerns about child sexual abuse. Child Abuse \& Neglect, 24(1), 149-157.

Paolucci, E. O., Genuis, M. L. \& Violato, C. (2001). A meta-analysis of the published research on the effects of child sexual abuse. The Journal of Psychology, 135(1), 17-36.

Pennington, N. \& Hastie, R. (1988). Explanation-based decision making: effects of memory structure on judgment. Journal of Experimental Psychology, 14(3), 521-533.

Pilatti, R. \& Silvino, A. M. D. (2009). Psicologia e deliberação legal no tribunal do júri brasileiro: proposição de uma agenda de pesquisa. Psicologia: Reflexão e Crítica, 22(2), 277-285.

Pilatti, R., Silvino, A. M. D., Miura, M. A. \& Caldas, L. S. (2010). Tribunal simulado: efeito da ordem das teses e do antecedente do réu. Paidéia, 20(46), $197-$ 206.

Pozzulo, J. D., Dempsey, J., Maeder, E. \& Allen, L. (2010). The effects of victim gender, defendant gender, and defendant age on juror decision making. Criminal Justice and Behaviour, 37, 47-63.

Realmuto, G. M. \& Wescoe, S. (1992). Agreement among professional about a child's sexual abuse status: interviews with sexually anatomically correct dolls as indicators of abuse. Child Abuse and Neglect, 16, 719-725.

Rovinski, S. L. R. (2009). Psicologia jurídica no Brasil e na América Latina: dados históricos e suas repercussões quanto à avaliação psicológica. Em S. L. R. Rovinski \& R. M. Cruz (Eds.). Psicologia jurídica: perspectivas teóricas e processos de intervenção (pp. 11-22). São Paulo: Vetor.

Rovinski, S. L. R. \& Cruz, R. M. (2009). Psicologia jurídica: perspectivas teóricas e processos de intervenção. São Paulo: Vetor.
Rovinski, S. L. R. \& Stein, L. M. (2009). O uso da entrevista investigativa no contexto da psicologia forense. Em S. L. R. Rovinski \& R. M. Cruz (Eds.). Psicologia jurídica: perspectivas teóricas e processos de intervenção (pp. 67-74). São Paulo: Vetor.

Sanderson, C. (2005). Abuso sexual em crianças: fortalecendo pais e professores para proteger crianças contra abusos sexuais e pedofilia. São Paulo: M Books do Brasil.

Santos, S. S. \& Dell'Aglio, D. D. (2008). Compreendendo as mães de crianças vítimas de abuso sexual: ciclos de violência. Estudos de Psicologia, 25(4), 595-606.

Stein, L., Pergher, G. K. \& Feix, L. F. (2009). Desafios da oitiva de criancas e adolescentes: técnica de entrevista investigativa. Programa Nacional de Enfrentamento da Violência Sexual Contra Crianças e Adolescentes. Subsecretaria de Promoção dos Direitos da Criança e do Adolescente. Secretaria Especial dos Direitos Humanos da Presidência da República. Childhood Brasil. Acessado em 27 de julho de 2010. Disponível em http://www.bancadigital.com.br/sedh/reader2/?p $\mathrm{ID}=3$.

Sternberg, R. J. (2000). Tomada de decisão e raciocínio. Em R. J. Sternberg (Ed.). Psicologia cognitiva (pp. 339-370). Porto Alegre: Artmed.

Tonetto, L. M., Kalil, L. L., Melo, W. V., Schneider, D. G. \& Stein, L. M. (2006). O papel das heurísticas no julgamento e na tomada de decisão sob incerteza. Estudos de Psicologia, 23(2), 181-189.

Trindade, J. (2004). Manual de psicologia jurídica para operadores do direito. Porto Alegre: Livraria do Advogado.

Tyler, K. A. (2002). Social and emotional outcomes of childhood sexual abuse: a review of recent research. Aggression and Violent Behavior, 7(6), 567589.

WHO - World Health Organization, Regional Office for Africa (2004). Child sexual abuse: a silent bealth emergency. AFR/RC54/15 Rev. 1. Obtido em 23 de julho de $2010 \quad$ de http:/ /afrolib.afro.who.int/RC/RC\%2054\%20Do c-En/AFR.RC54.15\%20Rev.1\%20Child. Sexual.Abuse.18.06.04-5a.pdf.

Recebido em: 17/05/2009 Reformulado em: 02/09/2010 Aprovado em: 01/11/2011 
Apoio: Conselho Nacional de Desenvolvimento Científico e Tecnológico - CNPq

Sobre as autoras:

Cátula Pelisoli é psicóloga pela Universidade do Vale do Rio dos Sinos - UNISINOS, especialista em Psicoterapia Cognitivo-Comportamental pela WP Centro de Psicoterapia Cognitivo-Comportamental, mestre e doutoranda em Psicologia pela Universidade Federal do Rio Grande do Sul - UFRGS. Atualmente, atua como psicóloga no Centro de Atenção Psicossocial Casa Aberta, é psicóloga clínica em Osório, Rio Grande do Sul e professora de cursos de graduação e pós-graduação da Faculdade de Ciências e Letras de Osório - RS. Também é integrante do Núcleo de Estudos e Pesquisas em Adolescência - NEPA UFRGS.

Lara Lages Gava é psicóloga pela Universidade Federal do Espírito Santo - UFES, mestre em Filosofia pela Universidade Federal do Rio Grande do Sul - UFRGS e doutoranda em Psicologia nesta mesma instituição. É funcionária do Instituto Geral de Perícias do Rio Grande do Sul, onde exerce o cargo de Perita Criminal/Psicóloga, atuando na realização de avaliações psicológicas investigativas em casos de suspeita de abuso sexual cometido contra crianças e adolescentes. É integrante do Núcleo de Estudos e Pesquisas em Adolescência - NEPA UFRGS.

Débora Dalbosco Dell’Aglio é psicóloga pela Pontifícia Universidade Católica do Rio Grande do Sul - PUCRS, mestre e doutora em Psicologia do Desenvolvimento pela Universidade Federal do Rio Grande do Sul - UFRGS. É docente do Programa de Pós-Graduação em Psicologia da UFRGS, orientadora de Mestrado e Doutorado e coordenadora do Núcleo de Estudos e Pesquisas em Adolescência - NEPA/UFRGS. 